\title{
Creation of twistless circles in a model of stellar pulsations
}

\author{
Andreea Munteanu ${ }^{\text {a }}$, Emilia Petrisor ${ }^{\mathrm{b}}$, Enrique García-Berro ${ }^{\mathrm{a}, \mathrm{c}}$ \\ \& Jordi José ${ }^{c, d}$ \\ ${ }^{a}$ Departament de Física Aplicada, Universitat Politècnica de Catalunya, Jordi \\ Girona Salgado s/n, Mòdul B-5, Campus Nord, 08034 Barcelona, Spain \\ ${ }^{\mathrm{b}}$ Departamentul de Matematica, Universitatea Politehnica Timisoara, Pta Regina \\ Maria 1, 1900 Timisoara, Romania \\ 'Institut d'Estudis Espacials de Catalunya, Edifici Nexus, Gran Capità 2-4, 08034 \\ Barcelona, Spain \\ ${ }^{\mathrm{d}}$ Departament de Física i Enginyeria Nuclear, Universitat Politècnica de \\ Catalunya, Av. Víctor Balaguer, s/n, 08800, Vilanova i la Geltrú (Barcelona),
} Spain

\begin{abstract}
In the present paper, we study the Poincaré map associated to a periodic perturbation, both in space and time, of a linear Hamiltonian system. The dynamical system embodies the essential physics of stellar pulsations and provides a global and qualitative explanation of the chaotic oscillations observed in some stars. We show that this map is an area preserving one with an oscillating rotation number function. The nonmonotonic property of the rotation number function induced by the triplication of the elliptic fixed point is superposed on the nonmonotonic character due to the oscillating perturbation. This superposition leads to the co-manifestation of generic phenomena such as reconnection and meandering, with the nongeneric scenario of creation of vortices. The nonmonotonic property due to the triplication bifurcation is shown to be different from that exhibited by the cubic Hénon map, which can be considered as the prototype of area preserving maps which undergo a triplication followed by the twistless bifurcation. Our study exploits the reversibility property of the initial system, which induces the time-reversal symmetry of the Poincaré map.
\end{abstract}

Key words: Stellar oscillations, low-dimensional chaos, nontwist maps 1991 MSC: 37E40, 37G15, 37E30

PACS: 97.10.Sj, 05.45.Pq, 95.10.Fh, 82.40.Bj, 05.45.Ac 


\section{Introduction}

During the past twenty years there has been increasing interest in the study of the coexisting regular and chaotic motions in Hamiltonian systems with few degrees of freedom. A basic problem of study was the transition to chaos in two degrees of freedom Hamiltonian systems which are slight perturbations of an integrable system, whose Hamiltonian is nondegenerate. In order to ease this task, most of the results on the dynamics of such Hamiltonian systems were obtained exploring the behaviour of a Poincaré map associated to a cross section in a fixed energy surface. A Poincaré map is an area preserving map (APM) defined on an annulus, that is, depending on $(r, \theta)$, where $r$ is a momentum-like coordinate and $\theta$ is an angular variable. Considering the lift of such a map, namely computing the angle without the restriction modulo $2 \pi$, one associates to an orbit $\left(r_{n}, \theta_{n}\right)$ the rotation number which is the limit:

$$
\rho=\lim _{n \rightarrow \infty} \frac{\theta_{n}-\theta_{0}}{2 \pi n}
$$

if it exists. If $\rho$ is an irrational number, then the orbit densely fills an invariant circle (KAM circle), while an orbit of rational rotation number $p / q$ - with $p, q$ coprime integers, $q>0$ - is a $q$-periodic orbit.

Typical questions of mathematical and physical interest include the persistence of the KAM circles after perturbation and determination of the threshold at which a circle of a given rotation number breaks up. The main results which answer these questions, such as the KAM theorem, the Poincaré-Birkhoff theorem, and the Moser twist theorem, are based on the validity of the twist property of the area preserving map transforming $(r, \theta) \mapsto\left(r^{\prime}, \theta^{\prime}\right)$, namely:

$$
\frac{\partial \theta^{\prime}}{\partial r} \neq 0, \forall r
$$

The twist condition implies the monotonic change of the rotation number $\rho$ with $r$. The APM satisfying this condition is called monotonic twist map or simply a twist map. During the last twenty years the monotonic twist APMs were studied extensively [1]. Consequently, their dynamics has gained almost complete understanding. In particular, this statement is a consequence of the late understanding that Hamiltonian systems have universal behaviour which can be revealed by studying explicit symplectic maps of the plane.

Recently, due to the increasing number of physical phenomena that are modeled by nontwist maps, this class of dynamical systems has finally captured the attention of the scientific community. The few studies on nontwist maps existent in the literature have revealed an unexpected variety of local and 
global bifurcations: dimerized island chains, periodic orbits collision, separatrix reconnection, vortex islands $[2,3,4]$.

In the present paper we study the Poincaré map associated to a periodic perturbation, both in space and in time, of a linear Hamiltonian system. The dynamical system originates from a model of stellar pulsations previously introduced in [5] for low mass stars $\left(M / M_{\odot}<8\right.$, where $M_{\odot}$ is the solar mass) and extended to intermediate mass stars $\left(M / M_{\odot} \in[8,11]\right)$ in [6]. The latter work included some preliminary results concerning the nontwist character of the map. Here, we show that this map is an APM with an oscillating rotation number function. In spite of the system being a perturbation of a linear Hamiltonian, we argue that due to both the acquired nontwist character and the periodic character of the perturbation, the map presents the typical features of a generic class of nontwist APMs, such as reconnection and meandering, with the nongeneric scenario of creation of vortices. The nonmonotonic property due to the triplication bifurcation is shown to be different from that exhibited by the cubic Hénon map, which can be considered as the prototype of APMs, and which undergoes a triplication followed by the twistless bifurcation. Our study exploits the reversibility property of the initial system, which induces the time-reversal symmetry of the Poincaré map. The paper is organized as follows. In $\S 2$ we introduce our model of stellar pulsations. Before getting into the detailed analysis of it, we consider worth recalling the most important features of nontwist maps. This is done in $\S 3$. In this section we will also emphasize the consequences of both the triplication bifurcation and of the symmetry properties. Later, in $\S 4$ we study the dynamics of our simple model of stellar pulsations. Finally in section 5 we summarize our major findings and we draw our conclusions.

\section{Description of the model}

Pulsating stars are perfect tests of the theory of stellar evolution since the comparison between the theoretical pulsational characteristics (periods, amplitudes, and growth time scales) and the observed ones can help substantially in the fine tuning of the stellar models. Low- and intermediate-mass stars are prone to experience recurrent thermal instabilities and substantial mass loss during certain intervals of their evolution. Moreover, a substantial amount of pulsating stars in the Galaxy shows irregular behaviour and this makes them interesting targets in the field of dynamics systems. The present model embodies the essential of stellar oscillations by assuming an oscillatory driving originating in the stellar interior in the form of sinusoidal pressure waves. This model belongs to the family of the so-called one-zone models which treat the star as a rigid core surrounded by a dynamic, homogeneous gas shell. The pressure waves propagate through a transition zone characterized by a certain 
transmission coefficient until they hit the stellar envelope and dissipate. No back reaction of the outer layers on the inner ones is considered. The dissipation of the pressure waves induces a fluctuation in the radius and velocity of the outermost layer and this variability makes the object of our study.

The variation of the interior radius, $R_{\mathrm{c}}$, around an equilibrium value, $R_{0}$, is given by $R_{\mathrm{c}}=R_{0}+\alpha R_{0} \sin \omega_{\mathrm{c}} \tau$, where $\tau$ is the time in years and $\alpha$ and $\omega_{\mathrm{c}}$ are the fractional amplitude and the frequency of the driving, respectively. In absence of any driving force, the equation of motion is

$$
\frac{d^{2} R}{d \tau^{2}}=\frac{4 \pi R^{2}}{m} P-\frac{G M}{R^{2}},
$$

where $M$ is the stellar mass and $R$ and $P$ are the radius and pressure in the shell of mass $m$, respectively. For convenience, we introduce the nondimensional variables $x \equiv R / R_{\star}-1$ and $t \equiv \omega_{\mathrm{m}} \tau$, where $R_{\star}$ is the equilibrium stellar radius and

$$
\omega_{\mathrm{m}} \equiv \sqrt{\frac{G M}{R_{\star}^{3}}}
$$

is the characteristic frequency of the star.

Considering that the additional perturbative acceleration is proportional to the driving acceleration with a proportionality coefficient $Q$ we obtain

$$
A=-\left.Q \frac{d^{2} R_{c}}{d \tau^{2}}\right|_{\text {ret }}
$$

and after some algebraic manipulation [6] the final equation of motion reads

$$
\begin{aligned}
& \dot{x}=y \\
& \dot{y}=-x-\epsilon \sin \left(\omega x-\omega t-\alpha \omega^{1 / 3} \sin \omega t\right)
\end{aligned}
$$

where the characteristic frequency of the system, $\omega$ results from the use of the dimensionless time unit and from the assumption that $R_{0}$ encompasses almost the entire stellar mass. It is defined as

$$
\omega \equiv \frac{\omega_{\mathrm{c}}}{\omega_{\mathrm{m}}}=\left(\frac{R_{0}}{R_{\star}}\right)^{-3 / 2} .
$$

In the action-angle coordinates $(r, \theta)$ resulting from the change $x=\sqrt{2 r} \cos \theta$ 
and $y=\sqrt{2 r} \sin \theta$, the Hamiltonian of the system is

$$
H(r, \theta)=r-\frac{\epsilon}{\omega} \cos \left(\omega \sqrt{2 r} \cos \theta-\omega t-\alpha \omega^{1 / 3} \sin \omega t\right) .
$$

The parameters of the system are $\epsilon$, the total driving amplitude, $\alpha$, the fractional driving amplitude and $\omega$, the characteristic frequency of the system. From the point of view of the parametric study, we take $\omega$ to be the most important parameter fixing the type of star to be studied and its evolutionary status. It is a measure of the ratio between the internal radius where the driving is produced and the equilibrium stellar radius. From the astrophysical perspective, we are interested in the dynamics of the system from Eq.(5) corresponding to $\omega \approx 3$, more exactly $\omega=3.0146$. This low value of $\omega$ is associated with evolved stages of intermediate mass stars, as it results from stellar evolutionary models $[7,8]$. Since we are interested in modeling the evolution

of this type of stars, we fix $\omega$ during our simulations. We have performed a thorough parametric study in the space $(\epsilon, \alpha)$ as it assures a fine tuning of the strength of the perturbation. As in [6], we are interested in $\epsilon<100 \%$ and $\alpha<40 \%$, which allow the perturbative approach.

\section{Nontwist maps}

The prototype of nontwist area preserving maps is the quadratic standard $\operatorname{map}[4,9]$ :

$$
\begin{aligned}
& r_{n+1}=r_{n}-k \sin \theta \\
& \theta_{n+1}=\theta_{n}+2 \pi \omega-r_{n+1}^{2}(\bmod 2 \pi) .
\end{aligned}
$$

The twist condition is violated along the curve $r=k \sin \theta$. For $k=0$ we get an integrable map, whose orbits lie on the circles of constant $r$. The rotation number function is $\rho(r)=\omega-r^{2} /(2 \pi)$. The circle $r=0$ has the maximum rotation number $\rho_{\max }=\omega$. It is called the twistless circle or shearless circle. A slight perturbation leads to the persistence of the twistless circle and nearby circles having a diophantine rotation number - that is, an irrational number badly approximated by rationals [10]. Hence, the nontwist standard map, defined above, has an invariant circle of maximum rotation number among the rotation numbers of the nearby orbits. For a fixed perturbation parameter $k$ and for $\omega$ chosen in such a way that the map has a pair of Poincaré-Birkhoff chains containing $p / q$-type periodic orbits, as $\omega$ varies the two chains approach each other and at a threshold $\omega=\omega_{r}(k)$ the hyperbolic points of the two chains enter the twistless circle. This is called the reconnection threshold [12]. At the 
(a)

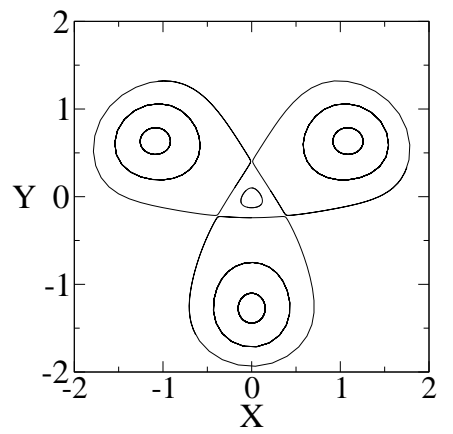

(b)

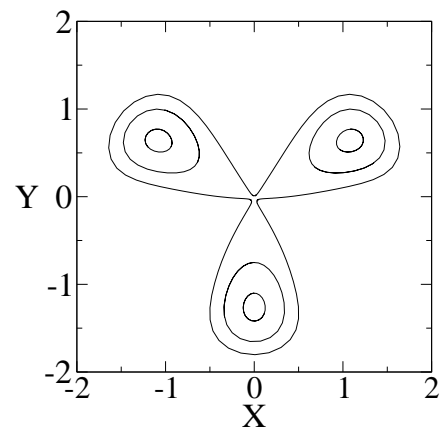

(c)

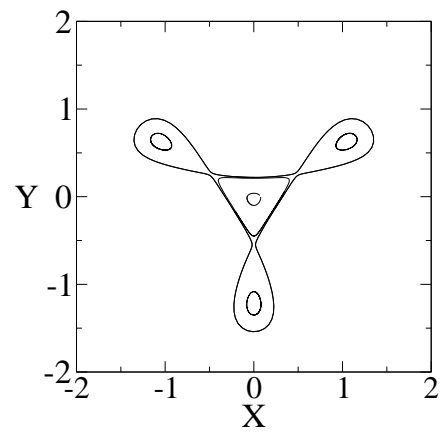

Fig. 1. The triplication scenario. The phase portrait around the elliptic point at the triplication threshold is shown in (b). Observe the change in the position of the heteroclinic triangle in (a) and (c).

reconnection threshold the two distinct hyperbolic orbits, of rotation number $p / q$, are connected by heteroclinic arcs.

There are many ways in which the monotonic twist condition can be violated. The vertical lines $\theta=\theta_{0}$ can be mapped into curves having a single extremum — quadratic twist [9] — or multiple extrema — cubic [11], quartic [12] or sinusoidal twist [13]. Moreover, it has been proved [14] that whenever the elliptic fixed point of an APM of the plane passes through a triplication, a twistless bifurcation occurs or, equivalently, the rotation number as a function of the distance from the elliptic fixed point becomes a nonmonotonic function. The triplication of the elliptic fixed point of an APM $f_{\mu}$ of the plane, occurs at the value $\mu \equiv \mu_{3}$ at which the multipliers of the elliptic point cross the values $\lambda=\exp ( \pm 2 \pi i / 3)$. At the triplication threshold $\mu=\mu_{3}$ an unstable period -3 orbit emerges from the elliptic fixed point in both directions, that is for $\mu<\mu_{3}$ and for $\mu>\mu_{3}$. As $\mu$ increases the elliptic point crosses the $1 / 3$ resonance and the triangular shape figure changes side after shrinking to elliptic fixed point (Figure 1).

The symmetry properties are very useful for the explanation of the behaviour exhibited by the system under study. We next recall some notions and results concerning time-reversal symmetry of a dynamical system [15]. A system of differential equations

$$
\dot{x}=F(x), x \in \mathbb{R}^{n},
$$

or equivalently, the vector field $F$ is called reversible if there is a smooth involution $R: \mathbb{R} \rightarrow \mathbb{R}(R \circ R=\mathrm{Id})$ such that $D R \circ F=-F \circ R$, where $D R$ is the differential of the map $R$. $R$ is called the reversor of the vector field $F$. If $\Phi_{t}$ is the flow of the vector field $F$, then the reversibility means that $\Phi_{-t} R=R \Phi_{t}$. This is tantamount to say that the reflection of the trajectory 
(a)

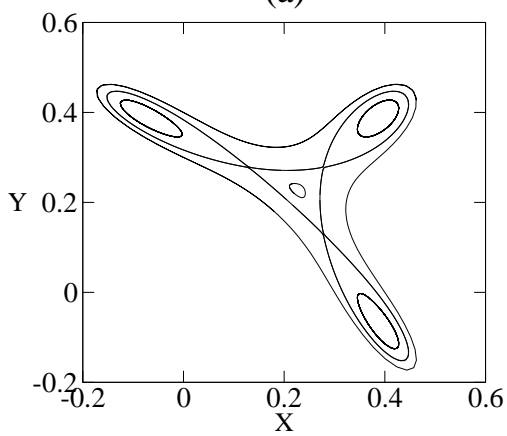

(b)

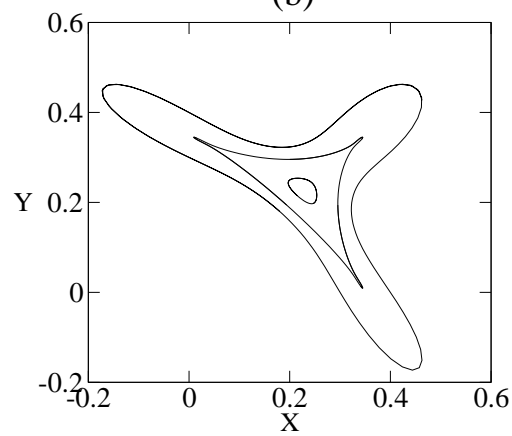

Fig. 2. The triplication bifurcation in the cubic Hénon map. (a) The separatrix associated to the hyperbolic period-3 orbit of the map from Eq.(11), corresponding to $\mu=-1.13$, and some nearby orbits; (b) The same map at the saddle-center threshold $\mu_{\mathrm{s}}=-1.12189$. The twistless circle is the circle with three cusps.

$x(t)=\Phi_{t} x_{0}$ is also an orbit of the system. A diffeomorphism $f: \mathbb{R}^{2} \rightarrow \mathbb{R}^{2}$ is called reversible with respect to a smooth involution $R$ of $\mathbb{R}^{2}$ if $f^{-1}=R \circ f \circ R$. If $f$ is an $R$-reversible diffeomorphism then $I=f \circ R$ is also an involution ( $I \circ I=\mathrm{Id}$ ), and $f=I \circ R$. This factorization of the map $f$ is very useful for the study of its dynamical properties. It can be shown that $f^{n}$ is a $R$-reversible map, too, $\forall n \in \mathbb{Z}$. Denote by $I_{n}=f^{n} \circ R, n \in \mathbb{Z}$, and by $\Gamma_{n}$ the fixed point set of the involution $I_{n}$. $\Gamma_{n}$ are called symmetry lines of the map $f$. A point in the intersection $\Gamma_{j} \cap \Gamma_{k}$ is a periodic point of the map $f$, whose period divides $|j-k|$. The symmetry lines $\Gamma_{k}$ are transformed by $f^{n}$ into other symmetry lines in the following way:

$$
f^{n} \Gamma_{k}=\Gamma_{2 n+k}
$$

In order to interpret the nontwist-type dynamics which will be encountered in our system when studying the triplication of the elliptic fixed point, we consider necessary to illustrate the twistless bifurcation in the case of the cubic Hénon map

$$
\begin{aligned}
& x_{n+1}=-y_{n}+x_{n}^{3}+\mu x_{n}+0.7 \\
& y_{n+1}=x_{n}
\end{aligned}
$$

which has an elliptic fixed point $\left(x_{0}, y_{0}=x_{0}\right)$ that can be easily found. At $\mu_{3}=-1.1505$ this point undergoes a triplication, while at $\mu_{\mathrm{s}}=-1.12189$ the saddle center collision occurs. In Figure 2, we show that as $\mu$ increases beyond $\mu_{3}$, the elliptic and hyperbolic period-3 orbits collide and disappear in a saddle-center bifurcation at $\mu=\mu_{\mathrm{s}}$. 


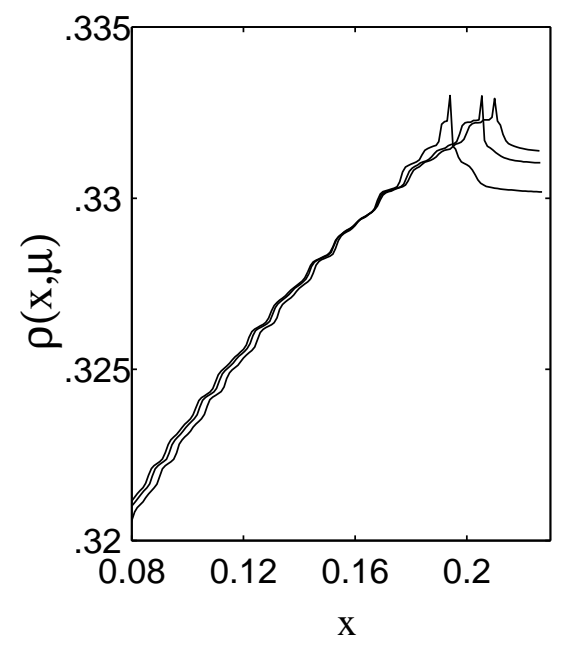

Fig. 3. Rotation number $\rho(x, \mu)$ versus $x$ for three values of $\mu$ between the triplication and saddle-center collision thresholds of the Hénon cubic map (Eq.11). The curves correspond to $\mu=-1.135, \mu=-1.13$ and $\mu=\mu_{\mathrm{s}}=-1.12189$. See text for more details.

In order to better identify the twistless bifurcation - the birth of a twistless circle - in Figure 3 we show the rotation number $\rho(x, \mu)$ as a function of the points $x$ lying on the symmetry line $\Gamma_{0}:=\{(x, y) \mid y=x\}$ of the cubic Hénon map, which is a reversible map with respect to the reversor $R(x, y)=(y, x)$. More precisely, the rotation number is computed at the points $x \in \Gamma_{0}$ and satisfying $x<x_{0}$. Figure 3 shows that between the triplication threshold and saddle - center collision threshold of the two period- 3 orbits, the twistless circle is the circle of rotation number $\rho_{\max }=1 / 3$ (the maximum points from right to left correspond to $\mu=-1.135, \mu=-1.13$, and $\left.\mu=\mu_{\mathrm{s}}=-1.12189\right)$. Observe that as $\mu$ increases up to $\mu_{\mathrm{s}}$, the twistless circle moves away from the elliptic fixed point.

Much more important (and difficult) is to detect the location of the twistless circle after the collision-annihilation of the two period- 3 orbits. In order to get some insight into the manifestation of the twist propertiy beyond the threshold $\mu_{\mathrm{s}}$, we have computed numerically the rotation numbers for points starting near the elliptic fixed point of the cubic Hénon map and on the symmetry line $\Gamma_{0}$. The rotation number functions $\rho(x, \mu)$ are plotted for $\mu=\mu_{\mathrm{s}}$, and some parameter values obtained varying $\mu$ in the direction given by the vector $v=\mu_{\mathrm{s}}-\mu_{3}$ (Figure 4 ). Note that the cubic Hénon map has a twistless circle of maximum rotation number among the rotation numbers of nearby orbits. Varying the parameter $\mu$ as specified above, $\rho_{\max }$ decreases as a result of the collision-annihilation of the orbits [4], and at some value, $\mu_{\mathrm{t}}$, the rotation number becomes decreasing. At this point the map is no longer nontwist. This is the reason why $\mu=\mu_{\mathrm{t}}$ was called the threshold of the twistless bifurcation in [14] where the above presented scenario was first presented although in the reverse order. 


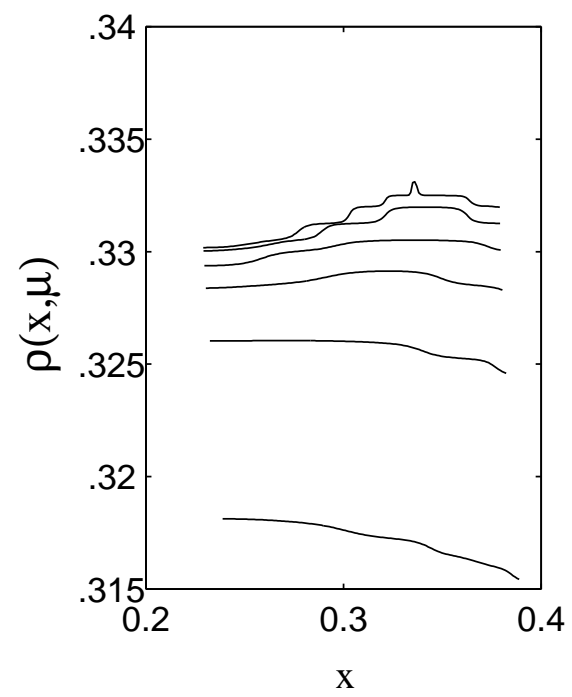

Fig. 4. Rotation number $\rho(x, \mu)$ of the points $(x, y)$ on the symmetry line $\Gamma_{0}$ of the map $f_{\mu}$, where $f_{\mu}$ is the Hénon map. From top to bottom: $\mu=-1.12189, \mu=-1.12$, $\mu=-1.115, \mu=-1.105, \mu=-1.08, \mu=-1$ and for $x>x_{0}$, where $\left(x_{0}, y_{0}\right)$ is the elliptic fixed point.

\section{Dynamical properties of the Poincaré map}

Due to the the complexity of the perturbative term of the Hamiltonian of Eq.(7), the associated system in Eq.(5) is analytically intractable. In order to get some insight into its dynamical behaviour, we study the Poincaré map (in fact the stroboscopic map) associated to this time-periodic Hamiltonian system. The unperturbed Hamiltonian $H_{0}(r, \theta)=r$ is globally degenerate, which means that $\partial^{2} H_{0} / \partial r^{2}=0, \forall r$. The dynamical consequence of this degeneracy is that all the orbits are periodic having the same period. This is in contrast to KAM theory, where the unperturbed system is nondegenerate. We will show that after a periodic perturbation (periodic in time and in space) the corresponding Poincaré map exhibits local and global bifurcations which in some cases are typical of the class of nontwist APMs which are perturbations of locally degenerate integrable APMs of an annulus, while in other cases are not.

\subsection{The nontwist property of the map}

Since the system given in Eq.(5) is time-periodic of period $T=2 \pi / \omega$, we define the function $s: \mathbb{R} \rightarrow \mathbb{S}^{1}, s(t)=t(\bmod 2 \pi / \omega)$, where $\mathbb{S}^{1}$ is the circle identified with the interval $[0,2 \pi / \omega)$. Hence, we have the autonomous system 
of differential equations:

$$
\begin{aligned}
& \dot{x}=y \\
& \dot{y}=-x-\epsilon \sin \left(\omega x-\omega s-\alpha \omega^{1 / 3} \sin \omega s\right) \\
& \dot{s}=1 .
\end{aligned}
$$

We denote by $\Phi_{t}^{\epsilon}$ its flow. $\Phi_{t}^{\epsilon}$ associates to each triplet $\left(x_{0}, y_{0}, s_{0}\right) \in \mathbb{R} \times \mathbb{S}^{1}$ the position at the time moment $t$ of the orbit starting at $t=0$ from $\left(x_{0}, y_{0}, s_{0}\right)$. The plane

$$
\Sigma=\left\{(x, y, s) \in \mathbb{R}^{2} \times \mathbb{S}^{1} \mid s=0\right\} \equiv \mathbb{R}^{2}
$$

is transversal to the flow and the map $P_{\epsilon}: \Sigma \rightarrow \Sigma$ defined by $P_{\epsilon}(x, y)=$ $\Phi_{2 \pi / \omega}^{\epsilon}(x, y, 0)$ is the associated Poincaré map.

It is a classical result in dynamical systems theory that the Poincaré map associated to a one degree of freedom periodic time-dependent Hamiltonian system is an APM. In order to interpret the dynamics displayed by the numerically computed Poincaré map, let us deduce some geometrical properties of this map. The vector field $F$, associated to the system from Eq.(12),

$$
F(x, y, s)=(y,-x-\epsilon \sin (\omega x-\omega s-b \sin (\omega s)), 1)
$$

with $\left(b=\alpha \omega^{1 / 3}\right)$, is reversible with respect to the involution $\mathcal{R}: \mathbb{R}^{2} \times \mathbb{S}^{1} \rightarrow$ $\mathbb{R}^{2} \times \mathbb{S}^{1}, \mathcal{R}(x, y, s)=(-x, y, 2 \pi / \omega-s)$, that is:

$$
F \circ \mathcal{R}=-D \mathcal{R} \circ F
$$

The reversibility property (15) is equivalent to $\mathcal{R} \circ \Phi_{-t}^{\epsilon}=\Phi_{t}^{\epsilon} \circ \mathcal{R}, \forall t \in \mathbb{R}$, which means that $\forall t \in \mathbb{R}$ the diffeomorphism $\Phi_{t}^{\epsilon}$ is $\mathcal{R}$-reversible, because $\Phi_{-t}^{\epsilon}=$ $\left(\Phi_{t}^{\epsilon}\right)^{-1}$. The Poincaré map associated to the vector field $F$ is $R$-reversible, with respect to the involution $R: \Sigma \rightarrow \Sigma, R(x, y)=(-x, y)$. Actually, notice that the reversor of the vector field $F$ is related to the involution $R$ by:

$$
\mathcal{R}(x, y, s)=(R(x, y), 2 \pi / \omega-s)
$$

It is clear that the definition of reversible maps is fulfilled by $P_{\epsilon}$ :

$$
\begin{aligned}
& P_{\epsilon}(R(x, y))=\Phi_{2 \pi / \omega}(-x, y, 0)=\Phi_{2 \pi / \omega}(\mathcal{R}(x, y, 2 \pi / \omega))= \\
& \mathcal{R} \Phi_{-2 \pi / \omega}(x, y, 2 \pi / \omega)=R P_{\epsilon}^{-1}(x, y) .
\end{aligned}
$$


(a)

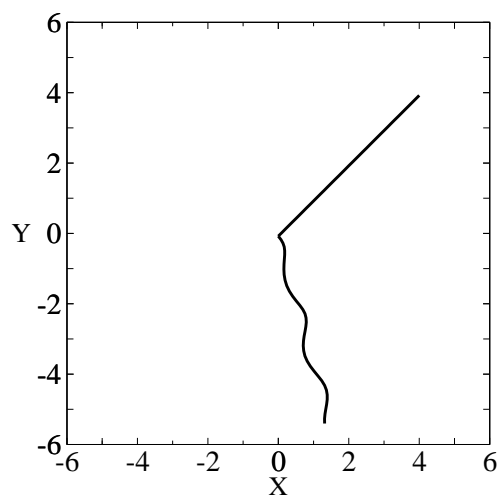

(b)

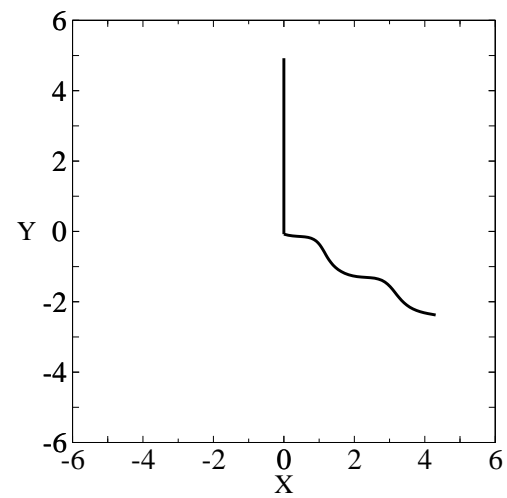

Fig. 5. The nontwist property of the Poincaré map. (a) The effect of the map on the semi-line defined by $\theta=\pi / 4$; (b) The effect of the map on the semi-line of $\theta=\pi / 2$.

The symmetry line useful in the analysis of the dynamical behaviour of the Poincaré map is $\Gamma_{0}=\operatorname{Fix}(R)$, having the equation $x=0$.

The Poincaré map $P_{0}$ has the elliptic fixed point $(0,0)$. After a slight perturbation the fixed point persists as a symmetric elliptic fixed point of the reversible map $P_{\epsilon}$ - it is a point $\left(0, y_{\epsilon}\right) \in \Gamma_{0} \cap \Gamma_{1}$. In order to reveal the nontwist property of the map $P_{\epsilon}$ we compute and visualize the effect of the map on different semi-lines emanating from the elliptic fixed point (Figure 5). As it results from Eq.(1), the rotation number for the map $P_{0}$ is negative, more exactly $\rho=-1 / \omega$. Note that unlike the standard-like nontwist maps, here a line of constant $\theta$ is not mapped onto a parabola, that is a curve with a single extremum, but onto an oscillating curve, having many minima and maxima. This fact will lead to a different behaviour of our nontwist map, in comparison to the dynamical properties of nontwist standard-like mappings.

Besides this primary way to illustrate the nontwist property of the map $P_{\epsilon}$, we computed the self-rotation number of the orbits of the map $P_{\epsilon}$, starting on the symmetry line $\Gamma_{0}$ and using the turning angle method [16]. The self-rotation number for the map corresponding to the parameter $\epsilon$ in the region of study is shown in Figure 6. Hence our map $P_{\epsilon}$ is a nontwist APM, whose rotation number function $\rho(y)$ is oscillating, having more than one extremum, that is, more than one invariant circle whose rotation number is a local extremum. Note that for the fixed parameter $\omega=3.0146$ of the Hamiltonian system of Eq.(5), the associated Poincaré map $P_{\epsilon}$ has an elliptic fixed point which undergoes triplication. In Figure 7 we represent the triplication curve $\alpha^{*}(\epsilon)$.

In order to emphasize the significant differences in the phase portraits before and after the triplication point, we add that in Figure 1 we illustrated the triplication bifurcation of the elliptic fixed point of the Poincaré map for $\omega=3.0146, \epsilon=0.07$ and $\alpha=-0.3-$ panel $(\mathrm{a})-\alpha=-0.05-$ panel $(b)$ 


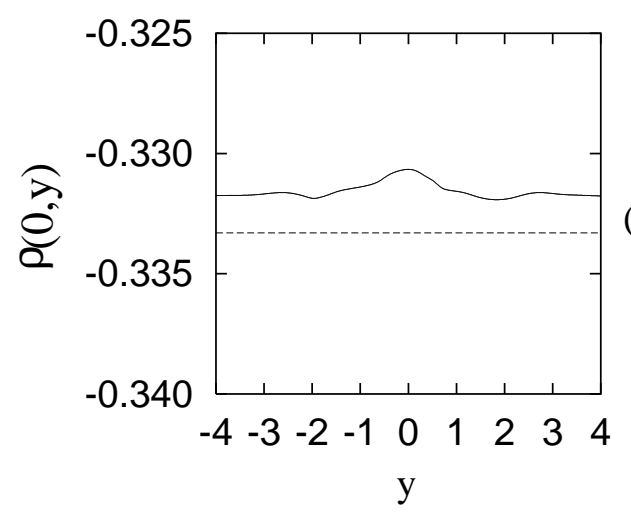

(a)

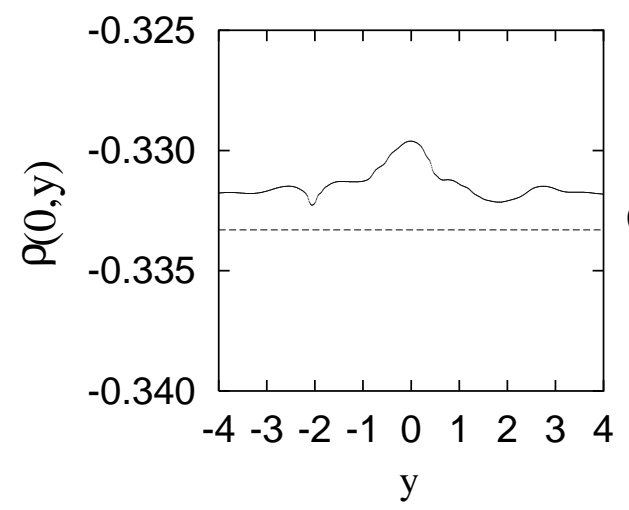

(b)

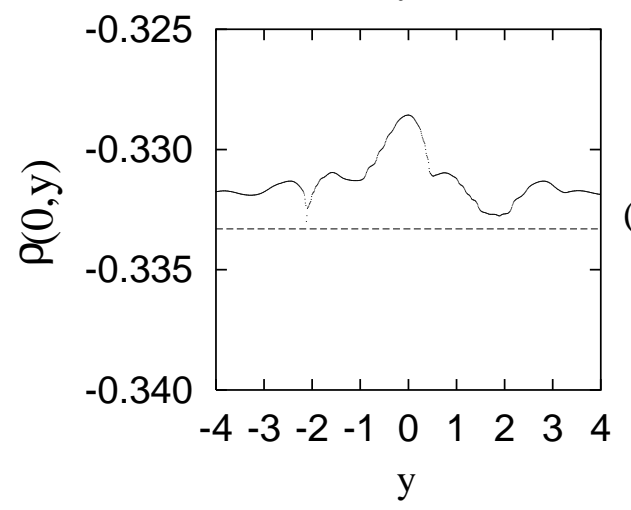

(c)

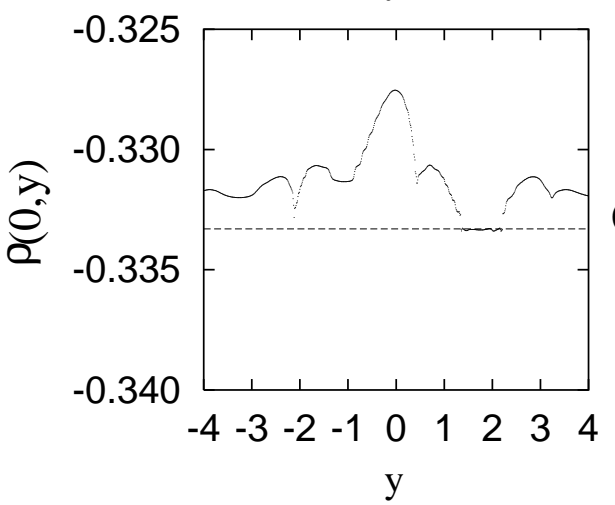

(d)

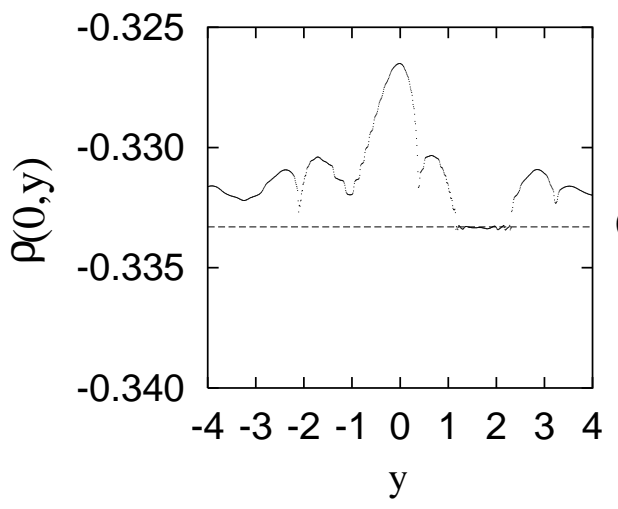

(e)

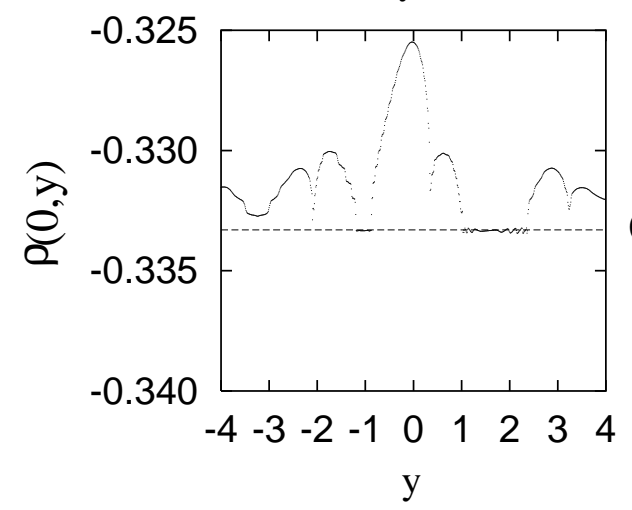

Fig. 6. The self-rotation number of the orbits of points $(0, y)$, with $y \in[-4,4]$. The horizontal axis is the axis of $y$-values, while the vertical one corresponds to the self-rotation number. The dashed line is at the level $\rho=1 / 3$. (a) $\epsilon=0.01$; (b) $\epsilon=0.02$; (c) $\epsilon=0.03$; (d) $\epsilon=0.04$; (e) $\epsilon=0.05$; (f) $\epsilon=0.06$.

- and $\alpha=0.02$ - panel (c). From the physical point of view it is only of interest the case in which $\alpha$ is constant and equal to $\sim 0.3$, and $\epsilon$ varies. Thus, the considered pairs $(\epsilon, \alpha)$ are located above the triplication curve. According to [14] for this case a twistless circle can exist. Comparing the rotation number associated to the cubic Hénon map (Figures 3 and 4) and our Poincaré map (Figure 6), we conclude that the Poincaré map has an oscillating rotation number with a larger amplitude near the elliptic fixed point. This could be interpreted as a superposition of the nontwist property induced by triplication on the nontwist behaviour due to the periodic perturbation of the initial Hamiltonian system. Near the elliptic fixed point, the Poincaré map has a neg- 


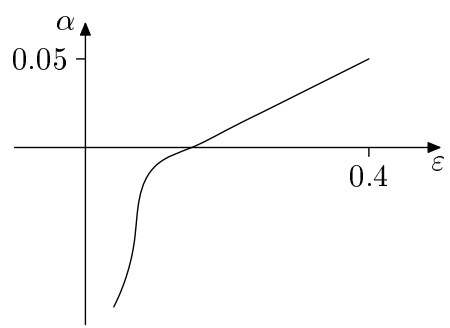

Fig. 7. The curve $\alpha^{*}(\epsilon)$ whose points are the triplication thresholds of the central fixed point of the map $P_{\epsilon}$.

ative twist. The apparent discontinuities of the rotation number are due to the distance chosen between the points on $\Gamma_{0}$ whose rotation number is computed. While on the branch $\Gamma_{0}^{+}=\{(x, y) \mid x=0, y>0\}$, a period-3 orbit has no point, it has one on the other branch, $\Gamma_{0}^{-}=\{(x, y) \mid x=0, y<0\}$. The orbit of a point on $\Gamma_{0}^{+}$encircling the homoclinic loops of the hyperbolic period-3 orbits can lead to an apparent discontinuity of the rotation number, although this function is continuous. The successive changes in the monotonicity of the map lead to a distinct nontwist behaviour in comparison with the known results reported for standard-like nontwist maps [4,9].

\subsection{Birth of the dimerized island chains. Local and global bifurcations}

In our parametric study, we first considered the cases in which $\epsilon$ is small, in order to study in detail the departure of the system from the simple harmonic oscillator as this parameter is increased. We restricted the study of the Poincaré map to a rectangle limited by initial conditions close to physically reasonable values of the radius and velocity of the outer stellar layers. We focused on the creation of a typical phase-space structure of nontwist maps: a pair of dimerized island chains. This characteristic scenario of creation of new orbits in nontwist maps is illustrated in Figure 8. Panel a exhibits the phase portrait of the near-to-integrable map, where the elliptic fixed point is surrounded by invariant circles. At $\epsilon \approx 0.03$ the Poincaré map has an invariant curve with three cusps (Figure $8 b$ ). Each cusp is a point of a new periodic orbit created through a saddle-center bifurcation for $\epsilon \approx 0.04$ : a stable and an unstable fixed point emanate from each cusp (Figure 8c). The homoclinic loop of every hyperbolic point surrounds the corresponding elliptic one. Between two successive hyperbolic points (in cyclic order) there is a heteroclinic connection. This separatrix structure called dimerized island chain appears in red in Figure $8 c$. In Figure $6 d$ we have shown the self-rotation number $\rho(0, y)$ for the case whose Poincaré map appears in Figure $8 c$. In the former figure, the plateau $\rho(0, y)=-1 / 3$ corresponding to $y \in(1,2)$ represents the constant rotation number due to the newly created elliptic fixed point on $\Gamma_{0}^{+}$. The 
(a)

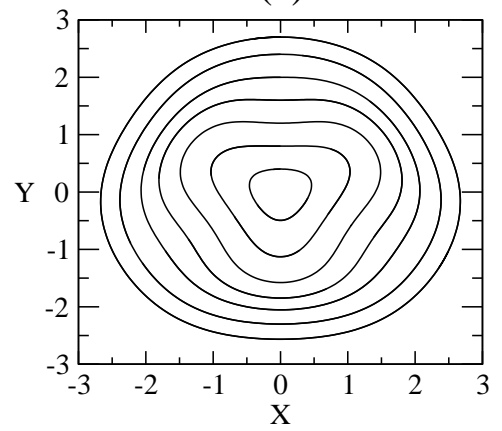

(c)

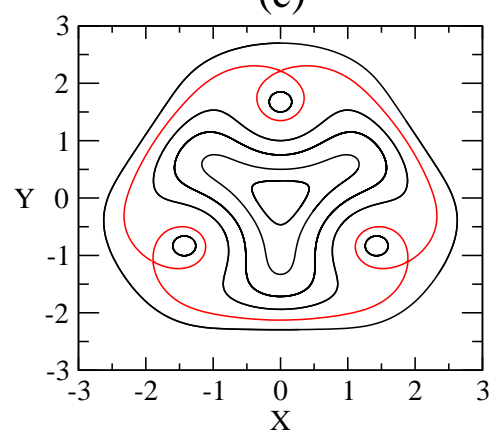

(b)

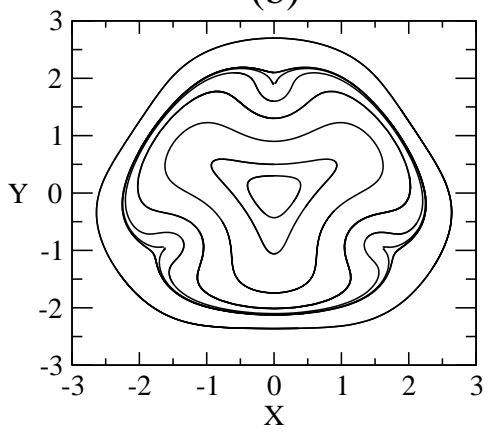

(d)

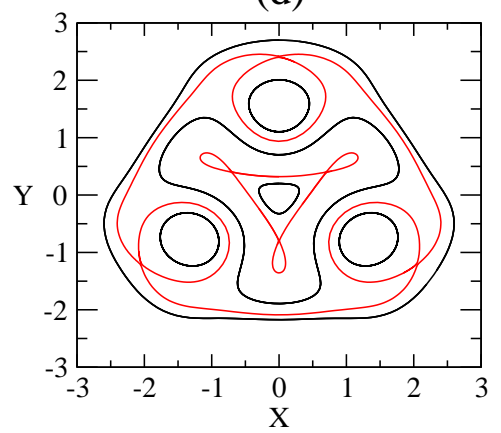

Fig. 8. The successive creation of the pair of dimerized island chains. Poincaré maps for $\alpha=0.3$ and different values of $\epsilon$ : (a) $\epsilon=0.01$ - an "almost" harmonic oscillator; (b) $\epsilon=0.03$ - the phase portrait just before the saddle-center bifurcation; (c) $\epsilon=0.04$ - the creation of the first dimerized island chain; (d) $\epsilon=0.07$ - the pair of period- 3 dimerized island chain is complete. The homoclinic and heteroclinic arcs of the island chains are shown in red.

dimerized island chain is also responsable for the minimum of $\rho(0, y)$ around $y \approx-2$.

Generically, dimerized island chains of the same period exist in pairs and are born in stages. The second period-3 dimerized island chain is created through the same bifurcation process at a higher value of $\epsilon$ (Figure 8d). In Figure 6f, the small plateau of $\rho(0, y)$ around $y \approx-1$ explains the birth of the second dimerized island chain. Between the two island chains the invariant circles are meanders, that is, the radius along such a circle is not a univoque function of the angle.

Around the last born period-3 dimerized island chain, a sequence of local and global bifurcations occurs. This is illustrated in Figure 9, where it can be seen that two independent orbits of the same period- 3 are created by saddlecenter bifurcation. They evolve in such a way that, finally, they interact with the orbits which belong to the first dimerized island chain. As $\epsilon$ increases the newly born elliptic points approach the hyperbolic points of the dimerized chain. When $\epsilon$ reaches a value of 0.11725 a global bifurcation occurs: the newly 


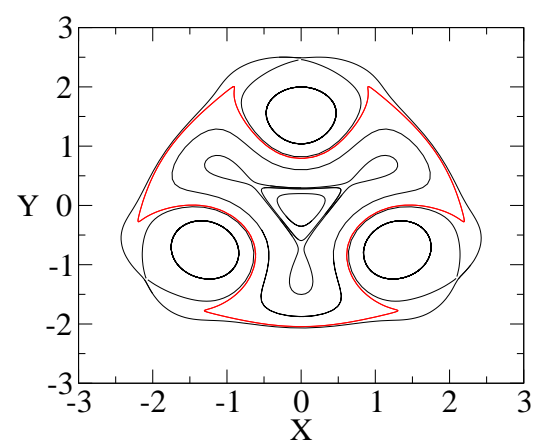

(a)

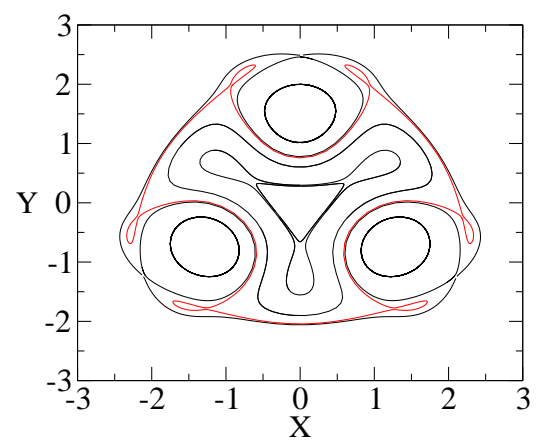

(b)

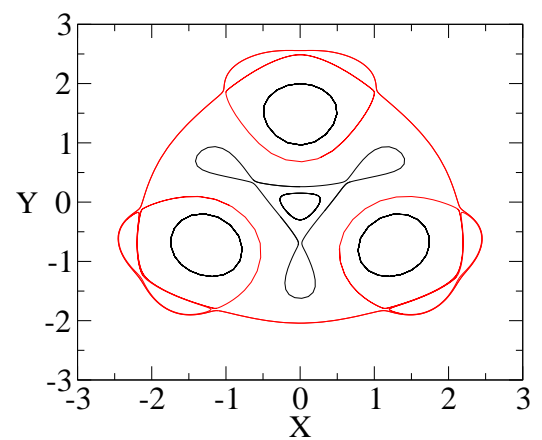

(c)

Fig. 9. The global bifurcation: creation by saddle-center bifurcations of a pair of period -3 orbits for $\alpha=0.3$ and increasing values of $\epsilon$. The particular orbits involved in the bifurcations appear in red. (a) $\epsilon=0.0875$ - the phase portrait at the threshold of a saddle-center bifurcation. It presents an invariant curve with six cusps; (b) $\epsilon=0.095$ - two interwined orbits of period -3 have been created. Inside each new-born loop there is a period-3 elliptic point; (c) $\epsilon=0.11725$ - the threshold of the global bifurcation. The topology of the separatrices has changed.

created orbits interfere, and the hyperbolic points of the dimerized island chain become hyperbolic points with homoclinic eight-like orbits encircling the newly created elliptic points.

In order to show the successive births of period-3 orbits as $\epsilon$ increases with $\alpha=0.3$ fixed, we compute and visualize $P_{\epsilon}^{k}\left(\Gamma_{0}^{+}\right)$and $P_{\epsilon}^{k}\left(\Gamma_{0}^{-}\right), k=1,2,3$. This process was first illustrated in Figure 5 a where $\Gamma_{0}^{+}$and $P_{\epsilon}^{1}\left(\Gamma_{0}^{+}\right)=\Gamma_{2}^{+}$ were represented. We show in Figure 10 the connection between the symmetry properties of the map and the creation of periodic orbits. A saddle-center 
(a)

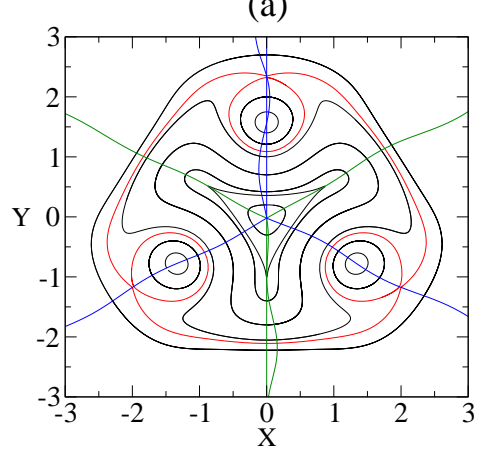

(b)

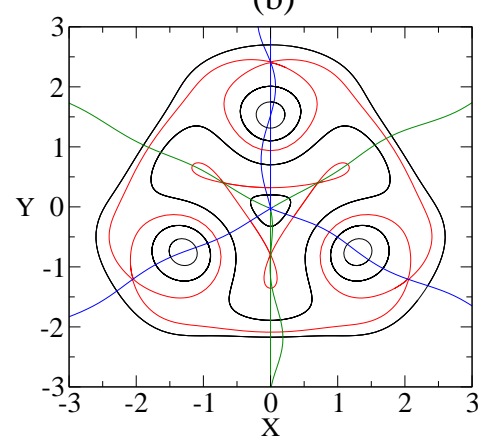

Fig. 10. The Poincaré map $P_{\epsilon}$ before and after a saddle-center bifurcation. The figures display also the symmetry lines $\Gamma_{2 k}^{+}$(in blue) and $\Gamma_{2 k}^{-}$(in green), with $k=0,1,2,3$. (a) $\epsilon=0.055$ - the phase portrait prior to the creation of the second dimerized island chain; (b) $\epsilon=0.07$ - the second dimerized island chain has been created. The points satisfying $(x, y) \in \Gamma_{0}^{ \pm} \cap \Gamma_{6}^{ \pm}$are period- 3 points.

bifurcation occurs at the value of the parameter $\epsilon$ at which $\Gamma_{6}^{ \pm}$has a tangential contact with $\Gamma_{0}^{ \pm}$. The corresponding Poincaré map has for such an $\epsilon$ an invariant curve with cusps which represent the points of tangency. Increasing the perturbation, the two symmetry lines intersect at two points, one being elliptic and the other, hyperbolic.

\subsection{Creation of chains of vortices}

A global bifurcation which is generic of nontwist maps is the so-called reconnection process, which is illustrated in Figure 11 for the nontwist multiharmonic standard map [4]:

$$
\begin{aligned}
& x_{n+1}=x_{n}+2 \pi \omega+y_{n+1}^{2} \quad(\bmod 2 \pi) \\
& y_{n+1}=y_{n}+k \sin \left[x_{n}+\arcsin \left(e \sin x_{n}\right)\right],
\end{aligned}
$$

with $k=0.2, e=0.38$ and different values of $\omega$. On the two sides of a twistless circle, two Poincaré-Birkhoff chains (necklaces of consecutive elliptic and hyperbolic points), having the same rotation number, approach each other (Figure 11a). At a given threshold, which is called the reconnection threshold, their hyperbolic points are connected by heteroclinic arcs (Figure 11b). Varying the parameter of the system further, two dimerized island chains emerge from the configuration created by the reconnection (Figure 11c). These chains are separated by meanders.

In our case, we witness the creation of the nongeneric vortices or dipoles [9]. Figure 12 displays the birth of the first chain of vortices. As $\epsilon$ increases, either 

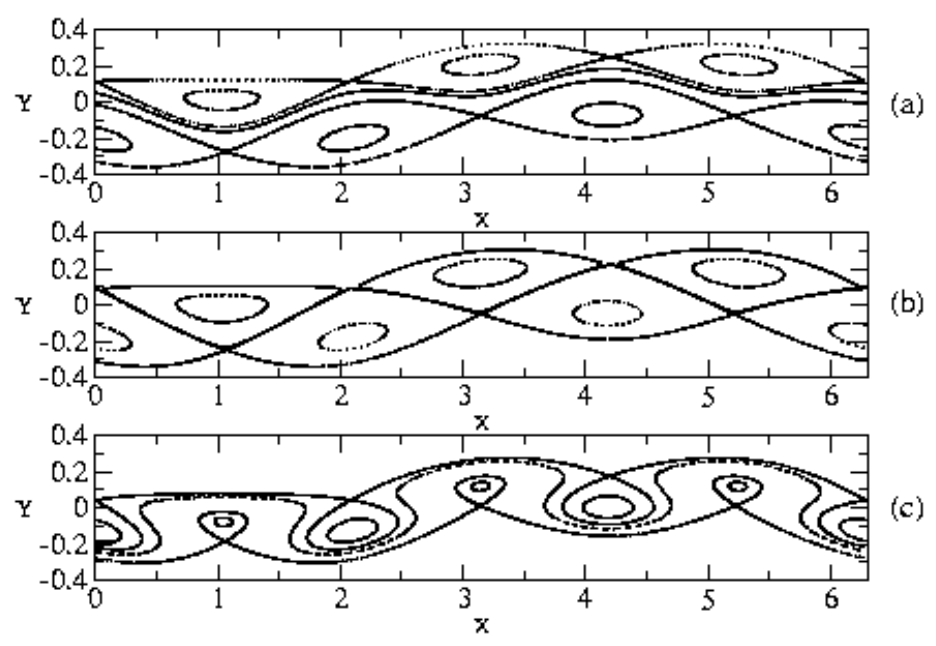

Fig. 11. Reconnection process in the nontwist standard-like multi-harmonic map. (a) $\omega=0.3287$ - two independent Poincaré-Birkhoff chains ; (b) $\omega=0.3297655$ — the reconnection threshold; (c) $\omega=0.3315$ — two dimerized island chains. We illustrate also a meander separating the two dimerized island chains.

$\Gamma_{6}^{+}$or $\Gamma_{6}^{-}$intersects tangentially at a new point located at a radius larger than in the previous cases. As a consequence, new period -3 dimerized island chains are created after $\epsilon$ crosses the value of tangential contact. However, each second dimerized island chain has a different creation mechanism. Unlike the previous case, the hyperbolic point of the first dimerized island chain bifurcates into two hyperbolic points in the transversal direction. In this process, a new elliptic point is born on the symmetry line. These hyperbolic points are connected by three heteroclinic arcs: one surrounding the previously existing elliptic point, another surrounding the new elliptic point, and the third one separating the two elliptic points. Thus, a pair of vortices is created.

As $\epsilon$ increases, the process of formation of chains of vortices continues for larger radii (Figure 13). Note that if the elliptic orbits of one chain of vortices intersect $\Gamma_{0}^{+}$, then the next one, which is created external to it, has a pair of elliptic points on the symmetry line $\Gamma_{0}^{-}$. The invariant rotational circle interpolating the hyperbolic points of the chain of vortices is the twistless circle. In Figure 13 it can be seen that such circles pass almost through the extremum of the symmetry lines $\Gamma_{6}^{ \pm}$. As already said, the formation of vortices is not generic and therefore the twistless circle that appears between them is not either.

\subsection{Formation of the stochastic sea}

Our system is a clear example of weak chaos, where the perturbation itself creates a separatrix network at a certain $\epsilon_{0}$ (the dimerized island chains and 
(a)

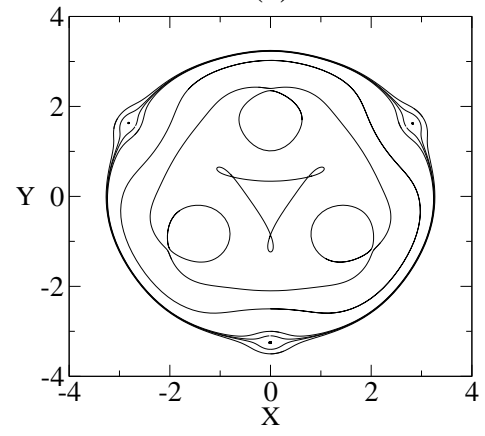

(b)

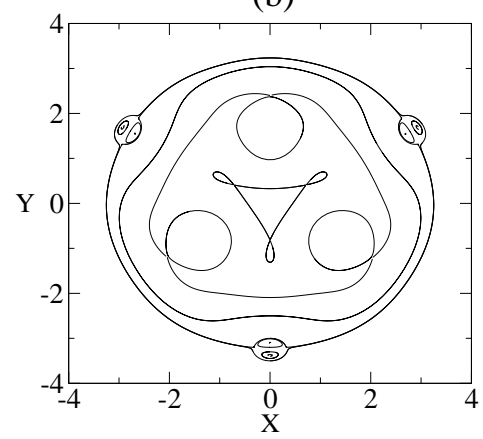

Fig. 12. Creation of the first chain of vortices for $\alpha=0.3$. (a) $\epsilon=0.0614$ The saddle-center bifurcation has created the first dimerized island chain; (b) $\epsilon=0.06617$ - instead of a second dimerized island chain, a pair of vortices is born. See text for more details.

the vortices chains) and then destroys it as $\epsilon$ increases by producing regions of chaotic dynamics. The case of strong chaos implies that the unperturbed Hamiltonian intrinsically has separatrix structures and the perturbation just clothes them in thin stochastic layers. In both cases, the merging of the stochastic layers in the phase space may give rise to a single chaotic network called stochastic web. The larger $\epsilon$ is, the wider is the stochastic web. Inside the cells of the web, there exists a set of islands of regular motion called invariant web-tori [17] whose dimensions are inversely proportional to the perturbation strength: for strong perturbations, they are engulfed by the stochastic sea. Our system presents this kind of behaviour, which is presented in Figure 14.

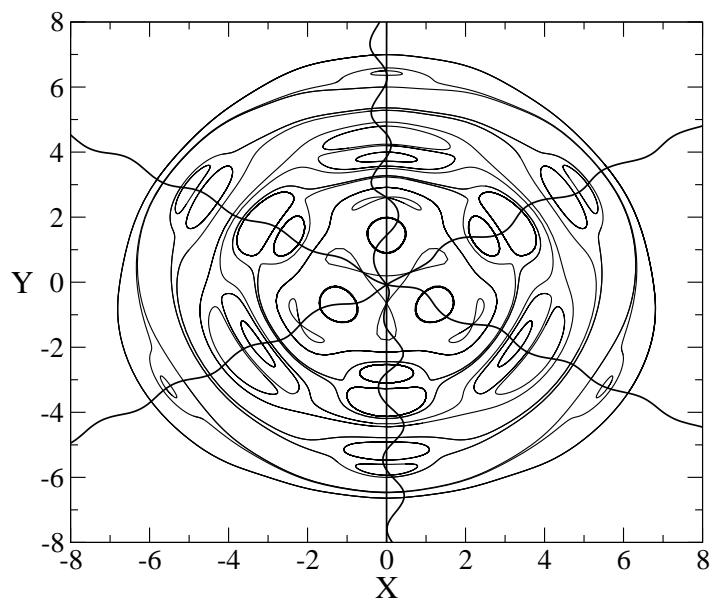

Fig. 13. The phase portrait of the Poincaré map corresponding to $\epsilon=0.2$ and $\alpha=0.3$. One can see a sequence of concentric chains of vortices. The most exterior one is still incomplete. The figure displays also the symmetry lines $\Gamma_{2 k}^{ \pm}, k=0,1,2,3$. 


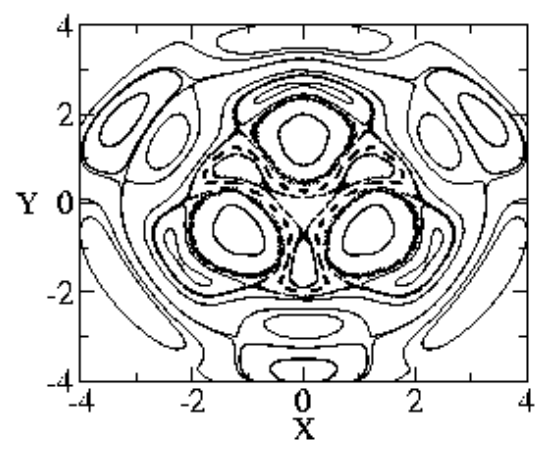

(a)

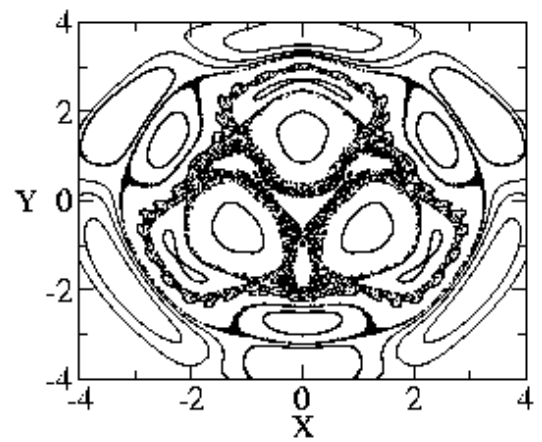

(b)

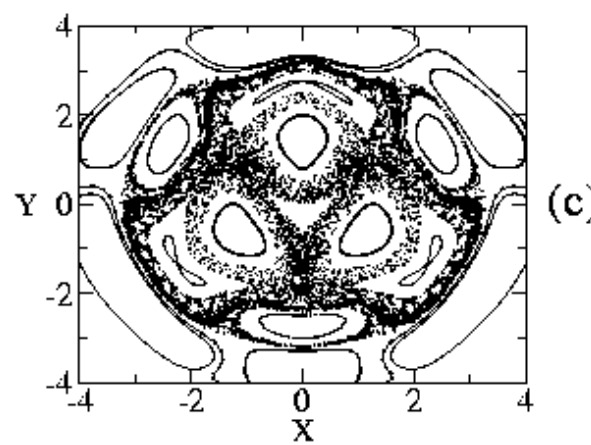

Fig. 14. Formation of the stochastic sea $(\alpha=0.3)$ : (a) $\epsilon=0.3-$ as the second dimerized island chain is destroyed, it is clothed in a stochastic layer; (b) $\epsilon=0.4$ - the stronger the perturbation, the wider the stochastic layers; (c) $\epsilon=0.5$ merging of the stochastic layers forming the stochastic sea.

It has long been known (and not yet well understood) that the boundaries of the islands of regular motion can be "sticky", meaning that chaotic orbits can spend a long time close to the islands and then escape in the chaotic sea. This phenomenon was observed already in [18] and was later called stickiness [19]. In general, it can be explained by the transformation of the web-tori into web-cantori as the perturbation is increased. Contrary to the absolute barriers constituted by the invariant tori, the cantori are only temporary barriers. That is, the orbits close to the main islands can reach the chaotic sea when they encounter the gaps in the cantori, which happens after an indefinite time interval. Recent works $[20,21]$ also suggest that the stickiness is due to the formation of higher-order resonant islands in the transition region between the main island 


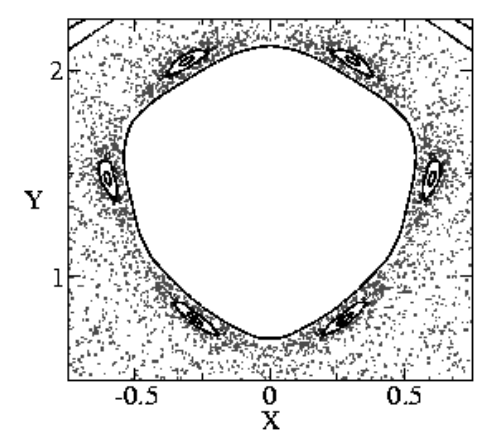

Fig. 15. A zoom of Figure $14 b$ illustrating the islands-around-islands hierarchy.

and the chaotic sea (Figure 15). The presence of these so-called dynamical traps appears to be generic for Hamiltonian systems and therefore their existence critically determines the large-timescale behaviour of such systems. In this context, a behaviour extremely similar to sticky orbits has been shown to result from simulations of stellar variability using full hydrodynamical codes carried on significantly long time-intervals. In the absence of a better explanation, it was attributed to long-term (secular) nonlinear effects in the stellar envelopes. In a previous work [6], we have shown that our simple model recovers such a behaviour as it is generic of Hamiltonian systems. We also presented clear examples of sticky orbits together with an extensive discussion of their implications in the framework of the classification of variable stars. Therefore, we mention here only the fact that if a Hamiltonian approach is intrinsic to the phenomenon of stellar variability, we can expect that different categories of variable stars (classified according to their amplitude, frequency, irregularity) may be viewed under a more unifying perspective than it is presently considered. Moreover, considering that the sporadic excursions of the sticky orbits to the chaotic sea translate into high velocities of the outer layers, mass loss is very likely to occur, again in accordance with the observations.

Another important and distinctive feature of the phase portrait of nontwist maps is the existence of meanders, as it was mentioned before when discussing Figure $8 d$. Meanders are created between two successively born dimerized island chains or between two chains of vortices. In nontwist standard-like maps they become usual invariant curves after the reconnection of the two chains. For the sake of clarity, we illustrate next the relationship between the meanders and the reconnection process for our map. In Figure 16 we show the creation of meanders from the reconnection of the Poincaré-Birkhoff chains of period 34. In the case of the standard nontwist map [9], in the reconnection of PoincaréBirkhoff chains of even periodic orbits, periodic points of the chains having the same stability type are aligned in phase. In other words, to an elliptic (hyperbolic) point of one chain corresponds below or above also an elliptic (hyperbolic) point. Due to this alignment, the reconnection is nongeneric and corresponds to a hyperbolic-hyperbolic collision leading to the formation of vortices. In our case, the two Poincare-Birkhoff chains of even period (period 
(a)

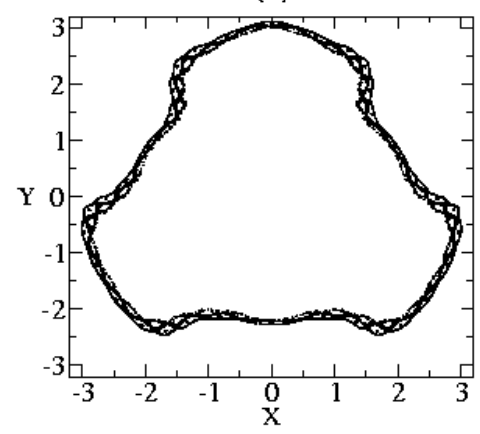

(b)

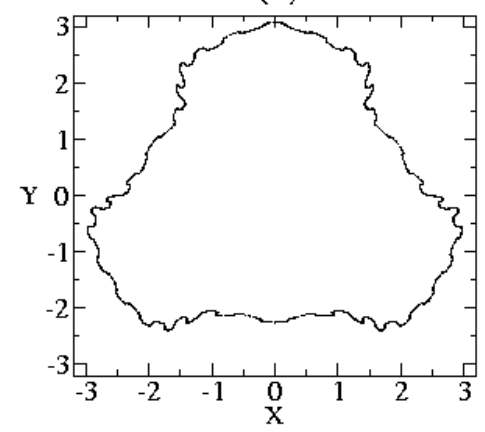

Fig. 16. The reconnection process: (a) $\epsilon=0.405$ - The Poincaré-Birkhoff chains confirming the generic process of reconnection illustrated in Figure 11; (b) $\epsilon=0.4$ - After the reconnection, the resultant meander with 34 cusps, slightly visible in Figure $14 b$.

34) have periodic points of opposite stability type which approach each other in their way to reconnection (Figure 16a). Therefore, the reconnection is generic and in the subsequent dimerized islands chains, hyperbolic-elliptic collision occurs. Moreover, after the reconnection, the twistless circle turns from a graph of a function of the angular variable to a meander (Figure 16b). This meander is slightly visible in Figure $14 b$.

Meanders appear to be robust invariant circles acting also as separatrices between distinct regions of chaotic dynamics. For stronger perturbations, their destruction allows the chaotic orbits to reach other stochastic regions previously separated by the meander. This behaviour was observed in nontwist standard-like maps [10], but until now there is no explanation for this robustness.

\section{Conclusions}

In the present work we have extended the preliminary results of Ref. [6] concerning the dynamics of a forced oscillator as a model of irregular stellar pulsations. The driving is characterized by two parameters, the fractional amplitude of the internal perturbation $\alpha$ and the total amplitude of the driving $\epsilon$. Our aim was to undertake a more detailed analysis of the transition to the nontwist property in the associated Poincaré map. We have demonstrated that for a given $\epsilon>0$ there exists an $\alpha^{*}(\epsilon)$ that corresponds to a triplication of the central elliptic fixed point of the map. As we were interested in values of the parameters $\alpha$ and $\epsilon$ above the triplication curve, the nontwist character of the map was undoubtly present [14].

Special attention was devoted to the process of formation of periodic orbits 
and to periodic-orbits collision. The reversibility of the Poincaré map and consequently, the symmetry properties allowed a clear identification of the structure of the periodic points. More precisely, the evolution and the intersections of the symmetry lines of the system provided good tracers of the steps of creation of dimerized island chains and vortex chains. We strongly argued in favor of these particularities of the map being entirely due to the superposition of two factors: the nonmonotonicity of the rotation number function induced by the triplication of the elliptic fixed point and the nonmonotonicity of the same function due to the oscillating perturbation. The latter feature admits alternative occurrence of maxima and minima, where the twist condition is violated. This translates into the minima and maxima of the symmetry lines which transform the invariant curves passing through them into twistless circles. Using these elements, we have followed the local and global bifurcations until the formation of stochastic layers around separatrices together with the associated sticky orbits. We have proved that the reconnection of even periodic orbits is generic by following the reconnection of two Poincaré-Birkhoff chains of period 34. From the astrophysical point of view, we have dwelled on the implications of the sticky regions and of the interplay between stochastic and regular regions in the framework of the analysis of stellar variability. In order to provide quantitative characterization of sticky orbits in the framework of stellar variability, we shall concentrate in the future on the causes and probabilistic estimates of the stickiness mechanism.

Acknowledgements. This work has been supported by the DGES grant PB981183-C03-02, by the MCYT grant AYA2000-1785, and by the CIRIT grant 1999SGR-00257. We also would like to acknowledge many helpful discussions with G.M. Zaslavsky.

\section{References}

[1] J.D. Meiss, Rev. Mod. Phys., 64, 795 (1992)

[2] D. del Castillo-Negrete, J.M. Greene, P.J. Morrison, Physica D, 100, 311 (1997)

[3] S. Shinohara, Y. Aikawa, Prog. Theo. Phys., 97, 379 (1997)

[4] E. Petrisor, Int. J. Bif. \& Chaos, 11, 497 (2001)

[5] V. Icke, A. Frank, A. Heske, A\&A, 258, 341 (1992)

[6] A. Munteanu, E. García-Berro, J. José, E. Petrisor, Chaos, 12, 332 (2002)

[7] C. Ritossa, E. García-Berro, I. Iben, Astrophys. J., 460, 489 (1996)

[8] E. García-Berro, C. Ritossa, I. Iben, Astrophys. J., 485, 765 (1997)

[9] D. del Castillo-Negrete, J.M. Greene, P.J. Morrison, Physica D, 91, 1 (1996) 
[10] C. Simó, Regular \& Chaotic Dyn., 3, 180 (1998)

[11] D. del Castillo-Negrete, M.C. Firpo, Chaos, 12, 496 (2002)

[12] J.E. Howard, J. Humpherys, Physica D, 80, 256 (1995)

[13] S. Saitô, Y. Nomura, K. Hirose, H. Ichikawa, Chaos, 7, 245 (1997)

[14] H.R. Dullin, J.D. Meiss, D. Sterling, Nonlinearity, 13, 203 (2000)

[15] J.S.W. Lamb, Physica D, 112, 1 (1998)

[16] H.R. Dullin, J.D. Meiss, D. Sterling, Physica D, 145, 25 (2000)

[17] G. Zaslavsky, R.Z. Sagdeev, D.A. Usikov, A.A. Chernikov, Weak chaos and quasi-regular patterns, Cambridge Univ. Press: Cambridge (1991)

[18] G. Contopoulos, Astron. J., 76, 147 (1971)

[19] R.B. Shirts, W.P. Reinhardt, J. Chem. Phys., 77, 5204 (1982)

[20] V. Rom-Kedar, G.M. Zaslavsky, Chaos, 9, 697 (1999)

[21] G. Zaslavsky, M. Edelman, B.A. Niyazov, Chaos, 7, 159 (1997) 